\title{
Military inventory capacity and stock planning with surge and warning time and supplier constraints
}

\author{
$\underline{\text { G. Calbert }}^{\text {a }}$, R. Thiagarajan ${ }^{\text {a }}$ and M. Rahman ${ }^{\text {a }}$ \\ ${ }^{a}$ Planning and Logistics, Decision Sciences Branch, Defence Science and Technology Group Edinburgh \\ $S A, 5111$. \\ Email: Greg.Calbert@dsto.defence.gov.au
}

\begin{abstract}
Linear programming has since its inception, always been strongly applied to inventory management. Linear programming has also been used extensively in many military stockage problems. In a military context, two general events test and shape the supply chain. The first is the development of inventory control: stockholding and infrastructure capacity policies to meet demands associated with ongoing raise, train and sustain requirements. In this case, the military inventory policies have very similar characteristics to stockholding in a commercial supply chain, where demands are forecasted and corresponding procurement takes place.
\end{abstract}

The second event that shapes the structure of the supply chain is the occurrence of a contingency demands, often termed a surge demand. In the second case, a contingency will have a planned demand, sustainment period and corresponding warning time. While the demand and sustainment period are critical to stockholding decisions, it is the warning time, which in conjunction with the vendors constraints on supplying at surge rates that is critical to developing inventory holdings, capacity and reserve stocks, often called war reserve materiel in the United States Department of Defence. One can often summarise the vendors constraints in supplying at a surge rate through the Contingency Provisioning Lead Time (CPLT), which is defined as that time the vendor can supply at the required contingency throughput rate. The CPLT may be greater than the warning time, indicating the vendor cannot supply at the required throughput rate at the start of the contingency.

With the basic input parameters of raise, train and sustain demands; contingency demand; warning time; CPLT; and maximum order quantities; we form a linear program that optimises the infrastructure capacity, reserve stocks and nominal inventory holdings at a Defence logistics installation. This linear program is extended to include an "anytime" contingency, where the inventory constraints are formed assuming contingency surge demand can occur at any time during the raise, train and sustain cycle.

The linear program is solved and sensitivity analysis is conducted over variation in CPLT and warning times. A further extension of the linear program is developed which includes constraints on bulk delivery lead times.

Finally, we discuss stochasticity in demand, supply and warning times. Inevitability, there is uncertainty in each of the aforementioned parameters. To address this, we discuss a two-stage stochastic linear program. In this construct, the first stage decisions are the optimal infrastructure capacity; raise, train and sustain inventory holdings; and reserve stock inventory holdings. The second stage variables are the procurement decisions required to ensure sustainability under different demand, supply and warning time scenarios. Approaches to solving the stochastic linear program exactly or approximately through the Monte-Carlo based stochastic average approximation algorithm are also discussed.

Keywords: Inventory management, military logistics, linear programming, stochastic linear programming 
Calbert et al., Military inventory capacity and stock planning with surge, warning time and supplier constraints

\section{INTRODUCTION}

Logistics infrastructure planning has always been critical as an enabler of Defence capability. Given that logistics supplies are the life-blood of Defence capability, developing and refining methods to examine logistics infrastructure are critical. Developing algorithms and methods that provide advice on logistics infrastructure requirements is the subject of this paper.

The mathematical optimisation technique of linear programming (LP) will be applied in this paper. While LPs have been extensively used in logistics planning for decades (Bixby, 2010), the contribution of this paper is in its application to Defence logistics requirements. Typically in Defence, logistics infrastructure is developed at particular locations based on distinct capability requirements. As an example, the logistics infrastructure at a particular location might be formed from the requirement to conduct raise, train and sustain (RTS) activities for an air-force squadron. Another example might be the formation of logistics infrastructure to meet a contingency requirement at some preparedness or warning time (Stevens and Ingram, 2013). The contribution made in this paper is to bridge the nexus between four distinct factors that shape logistics infrastructure requirements.

The first factor is cost minimisation. Planned infrastructure should meet Defence's requirements, whilst minimising costs. The second factor is to meet baseline logistics requirements. Such requirements are ongoing and include the aforementioned RTS activities at a particular location. The third factor is contingency logistics requirements. Defence plans different contingencies with an associated preparedness requirement which is specified as a warning time. For some contingencies, Defence should have the logistics capability to respond with essentially zero warning time, for example humanitarian and disaster relief operations. Others require a considerable work-up time, where significant warning is given. The final factor required in the design of Defence logistics infrastructure is resilience. Defence should be able to logistically respond with supplies, even in the event of upstream supply chain failures.

This paper is structured as follows. Section 2 outlines the fundamental differences between Defence and civilian supply chains. Section 3 describes the modelling framework in forming a LP formulation for infrastructure capacity, holdings and reserves required to meet Defence demands. In Section 4 we discuss the formulation of the LP and develop an example. Section 5 introduces uncertainty in each of the modelling inputs and discusses a stochastic linear program specifying infrastructure requirements. The final Section 6 discussions future research directions.

\section{CIVILIAN AND DEFENCE LOGISTICS SUPPLY CHAINS}

For most supplies, such as fuel, food and medical supplies, Defence sources from the civilian supply chain. Nonetheless, at what is termed the point of demand, the characteristics of Defence demands are quite different from that of typical civilian supply chains. When compared to Defence demands, civilian demands can be quite predictable and seasonal in nature. For civilian supply chains it is cost and reliability that are key forces shaping contracting decisions on supply. For Defence, logistics contracting must be shaped by reliability, resilience and then cost. Here, one should distinguish between reliability, and resilience. A reliable supply chain ensures that demands can met be met under most commercial circumstances. Resilience implies that demands can be met, even in the event of an upstream supply chain blockage. Table 1 juxtaposes the two supply chains at the point of demand.

Table 1: Differences between civilian and defence supply chains at the point of demand.

\begin{tabular}{|l|l|}
\hline Civilian & Defence \\
\hline Moderate to high predictability of demands & Very low to moderate predictability of demands \\
\hline No reserves, only cycle stock and safety stock & Reserves, if required, plus cycle stock and safety stock. \\
\hline Reasonability smooth seasonal transitions in demand & Spike demands with variable warning time \\
\hline The cost then reliability but not resilience are key drivers & The reliability, resilience, and then cost are the key drivers \\
\hline
\end{tabular}

Defence does not require that its logistics supply chains meet all demands under all circumstances. Instead, a key pillar of defence logistics planning is the notion of preparedness or warning time. Defence should form its logistics infrastructure to meet ongoing RTS demands. Over and above these RTS demands; defence also specifies preparedness warning times (hitherto known as just warning time) for different scenario demands at particular locations (Defence, 2004). The length of the warning time will depend on the scenario. In turn, defence cannot demand from civilian supply chains unlimited throughput under all circumstances. Instead 
Calbert et al., Military inventory capacity and stock planning with surge, warning time and supplier constraints

Defence bases its supply chain plans from a contingency provisioning lead time, which is defined as that time

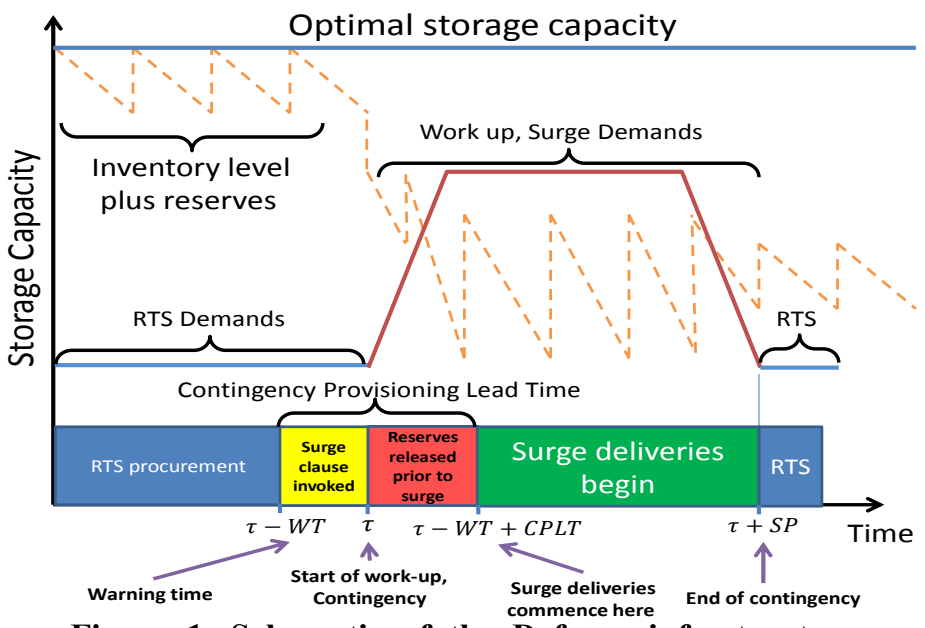

Figure 1. Schematic of the Defence infrastructure optimisation problem. required by industry to supply at the required rate specified by scenario demands. Such a demand rate is termed the surge. Again, depending on the logistics item, the contingency provisioning lead time (CPLT) will vary from a period of days, or many months. It is the interplay between the warning time and the CPLT that is a strong determinant of the logistics infrastructure and item storage required. This is illustrated schematically in Figure 1.

While logistics infrastructure is taken as a system of warehouses and transport to meet capability demands, in this paper we refer to such infrastructure as the capacity of the defence supply chain node. The capacity will be measured either as units of pallets, or cubic metres, depending on the item. Thus the optimisation problem that we solve is to minimise the capacity, inventory holdings and reserve stocks, required for a contingency, ensuring both RTS demands, along with contingency demands (surge) are met, subject to the supply chain constraints of lead time, maximum order quantities, warning times, and CPLT. Reserve stocks are assumed only to be used to cover the supply short fall of nominal RTS supplies prior to the CPLT, when supplier surge commences. By minimising the capacity, we intrinsically minimise the costs. Given this background we now form the mathematical model.

\section{THE MODELLING FRAMEWORK}

The input and decision variables for the optimisation problem are defined in Table 2.

Table 2: Decision variables of the linear program.

\begin{tabular}{|c|c|c|}
\hline Variable & Notation & Type \\
\hline Time and time horizon & $t=1, \cdots, T$ & Input \\
\hline RTS demand time series & $d_{1}, d_{2}, \cdots, d_{T}, d_{t} \geq 0, t \in\{1, \cdots, T\}$ & Input \\
\hline Warning or preparedness time & $W T \geq 0$ & Input \\
\hline Sustainment period of the contingency & $S P>0$ & Input \\
\hline Surge demand time series & $c_{1}, c_{2}, \cdots, c_{S P}, \quad c_{j} \geq 0, j \in\{1, \cdots, S P\}$ & Input \\
\hline Maximum order quantity & $d_{\max }$ & Input \\
\hline Maximum surge quantity & $s_{\max }$ & Input \\
\hline Lead time for deliveries & $L$ & Input \\
\hline Contingency provisioning lead time & CPLT & Input \\
\hline Time the contingency starts & $\tau \in\{1, \cdots, T\}$ & Input \\
\hline $\begin{array}{l}\text { Inventory level at the cycle top at time } t \\
\text { given the contingency starts at time } \tau\end{array}$ & $I_{t}^{t o p}(\tau), \quad t, \tau \in\{1, \cdots, T\}$ & Decision \\
\hline $\begin{array}{l}\text { Inventory level at the bottom of the cycle at } \\
\text { time } t \text { given the contingency starts at time } \tau\end{array}$ & $I_{t}^{\text {bottom }}(\tau), t, \tau \in\{1, \cdots, T\}$ & Decision \\
\hline Initial inventory & $I_{0}$ & Decision \\
\hline Infrastructure capacity & $C$ & Decision \\
\hline Reserve stock & $R$ & Decision \\
\hline Nominal RTS procurement & $x_{1}, x_{2}, \cdots, x_{T}, x_{t} \geq 0, t \in\{1, \cdots, T\}$ & Decision \\
\hline Surge procurement & $s_{1}, s_{2}, \cdots, s_{S P,} \quad s_{j} \geq 0, j \in\{1, \cdots, S P\}$ & Decision \\
\hline
\end{tabular}


Calbert et al., Military inventory capacity and stock planning with surge, warning time and supplier constraints

Time is modelled as days as is the warning time (WT), contingency sustainment period (SP) and CPLT. Normal RTS deliveries occur until surge deliveries commence. While normal deliveries occur, during the warning time, the logistics vendor is contacted to invoke the surge deliveries. Because the contingency (which for the purpose of clarity includes work-up operations to support the contingency) is assumed to start

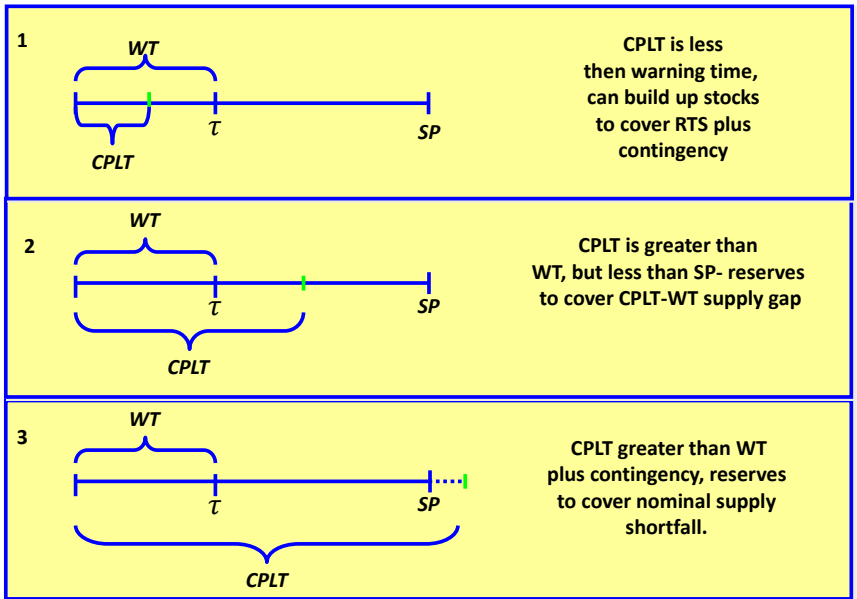

Figure 2. Three cases covering different CPLT, WT and SP values at time $\tau$ the warning occurs at time $\tau-$ $W T$, thus the time that the surge deliveries begin is $\tau-W T+C P L T$.

Now there are three cases to consider in the analysis. First the CPLT is less than the warning time; $C P L T \leq W T$. In this case, the surge deliveries can begin at the start of the contingency. Reserve stocks may still be required, but only to cover the shortfall between the surge deliveries and the contingency demands. Second the CPLT is greater than the warning time. In this case, surge deliveries start after the commencement of the work-up and contingency demands. Assuming normal RTS supply maxima cannot match contingency demands; reserves must cover the time-gap, CPLT $-W T$, between the start of the contingency and the first delivery of the surge. Finally, if the CPLT

is greater than the warning time plus the sustainment period then reserve stocks must supplement normal RTS supply alone, to meet the contingency requirements.

Now some notation is introduced. Let $t_{s}(\tau)$ be the time that the surge deliveries commences, that is $t_{s}(\tau)=$ $\max (\tau, \tau-W T+C P L T)$.

Here we assume that if the surge can occur prior to start of the work up, contingency, then this surge throughput is still timed to meet the start of the work-up, contingency. It is also assumed that $t_{S}(\tau)<\tau+S P$ so surge deliveries are actually possible. If not, then a slightly different approach sees only normal deliveries and reserves covering contingency demands. Similarly, the end of the surge is termed $t_{e}(\tau)$ with $t_{e}(\tau)=$ $\min (\tau+S P, T)$. This equation simply states that if the contingency sustainment period extends beyond the planning horizon, the last surge delivery is modelled at the planning horizon. The planning horizon, $T$ can easily be extended so this situation does not happen.

Having defined these equations we are now in a good position to define the inventory level over all times, $t$ based on $t_{s}(\tau)$ and $t_{e}(\tau)$. Here again there are several circumstances. When $t<\tau$, that is prior to the start of the work-up and contingency, the flow of fuel is as for normal RTS, thus the same inventory dynamics for the top and bottom of the cycle apply. No reserve stocks are released at this stage. When $\tau \leq t<t_{s}(\tau)$ the work-up and the contingency have started but the surge has not, so the reserves are released to cover the shortfall in demand. When $t_{s}(\tau) \leq t<t_{e}(\tau)$ then both surge and reserves cover the work-up, contingency demands. Finally, when $t_{e}(\tau) \leq t \leq T$ normal RTS supply re-commences after the contingency ends.

We have to define the bottom and top of the inventory cycle, which will be depend on the start of the contingency, which we label as $I_{t}^{\text {bottom }}(\tau)$ and $I_{t}^{\text {top }}(\tau)$. Assuming supplies can be delivered on the day of ordering $(L=0)$, the bottom of the cycle, which is the inventory level just prior to a delivery has the form

$$
I_{t}^{\text {bottom }}(\tau)=\left\{\begin{array}{c}
I_{o}-\sum_{i=1}^{t} d_{i}+\sum_{i=1}^{t} x_{i}, t<\tau, \\
I_{0}+R-\sum_{i=1}^{\tau-1} d_{i}-\sum_{i=\tau}^{t} c_{i-\tau+1}+\sum_{i=1}^{t} x_{i}, \tau \leq t<t_{s}, \\
I_{0}+R-\sum_{i=1}^{\tau-1} d_{i}-\sum_{i=\tau}^{t} c_{i-\tau+1}+\sum_{i=1}^{t_{s}-1} x_{i}+\sum_{i=t_{s}}^{t} s_{i-t_{s}+1}, t_{s} \leq t<t_{e}, \\
I_{0}+R-\sum_{i=1}^{\tau-1} d_{i}-\sum_{i=\tau}^{t_{e}} c_{i-\tau+1}-\sum_{i=t_{e}+1}^{t} d_{i}+\sum_{i=1}^{t_{s}-1} x_{i}+\sum_{i=t_{s}}^{t_{e}} s_{i-t_{s}+1}+\sum_{i=t_{e}+1}^{t} x_{i}, t_{e} \leq t \leq T .
\end{array}\right.
$$

If there is a non-zero lead time, (which is distinct from the CPLT), $L$ to deliver the logistics items, $x_{i}$ and $s_{i}$ are simply replaced by $x_{i-L}$ and $s_{i-L}$ respectively in the expression for $I_{t}^{\text {bottom }}(\tau)$. Though the above 
Calbert et al., Military inventory capacity and stock planning with surge, warning time and supplier constraints

expression might look complicated, equation (1) is merely materiel inflows and outflows at the aforementioned stages of normal RTS supply, RTS supply plus reserves, surge supply plus reserves, then the return to RTS supply post-contingency. The expression for $I_{t}^{t o p}(\tau)$ has a similar form in that the top of the cycle is the inventory level just after a delivery. For example, the first term for $I_{t}^{\text {top }}(\tau)$, when $t<\tau$ excludes the demand at time $t$, that is $I_{o}-\sum_{i=1}^{t-1} d_{i}+\sum_{i=1}^{t} x_{i}$.

Having formulated what are the bottom and top of the inventory cycle, the next step is to form the LP.

\section{THE LINEAR PROGRAM FOR AN ANYTIME CONTINGENCY}

While the RTS fuel demand pattern will broadly occur in a training cycle, a contingency and its associated work-up activities can occur at any time. The LP with such an anytime contingency induces a large number of constraints. For each inventory level calculation at time $t$ the contingency can occur at any $\tau \in\{1, \cdots, T\}$. For equation (1), there will $2 T$ constraints, one each for the top and bottom of the cycle at each time. For each $\tau$ (the time that the work-up and the contingency commences) there are $T$ constraints. Therefore there are $2 T^{2}$ inventory constraints and $T+S P$ constraints ensuring the orders do not exceed the maximal order quantities. Finally there are $T+S P+3$ constraints to ensure positivity of the variables. Therefore, the structure of the LP looks like this

$$
\begin{gathered}
\min _{I_{o}, C, R, x_{1}, \cdots, x_{T}, s_{1}, \cdots, S_{S P}} C+R \\
I_{t}^{\text {bottom }}(\tau)>0, \forall t, \tau \in\{1,2, \cdots, T\} \\
I_{t}^{\text {top }}(\tau) \leq C-\mathbf{1}_{t<\tau} R, \forall t, \tau \in\{1,2, \cdots, T\} \\
x_{t} \leq d_{\max }, \forall t \in\{1,2, \cdots, T\}, \\
s_{i} \leq s_{\max }, \forall i \in\{1, \cdots, S P\}, \\
I_{0}, C, R, x_{1}, x_{2}, \cdots, x_{T}, s_{1}, \cdots, s_{S P} \geq 0 .
\end{gathered}
$$

The indicator function $\mathbf{1}_{t<\tau}$ which is one if $t<\tau$ and zero otherwise, in the second constraint for the top of the inventory cycle ensures that prior to the start of the contingency $(t<\tau)$ the top of the inventory cycle is not so large that the reserves cannot be stored within the infrastructure capacity.

\subsection{An example}

As an example of the interplay between the CPLT and the WT, consider the following example based on the supply of fuel to an air force fuel
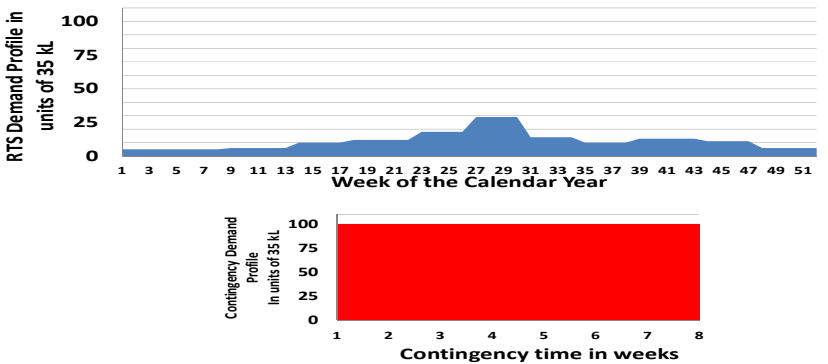

Figure 3: RTS and contingency demands used in the example. installation. Orders placed on any given day are delivered on that day so $L=$ 0 . The supply chain can be considered as a flow of fuel from the fuel industry terminal to the air force base. In other examples, the fuel vendor may place constraints on the minimal order size and there may be significant lead times to receive such orders, because they are shipped. There is also the added complexity for fuel that delivery timing will also be determined by the tank structure. Generally fuel deliveries require an empty on-receipt quality control tank. All the aforementioned complexities can be incorporated in the above framework though the addition of extra constraints.

At a particular location, an industry vendor can deliver $23 \times 35$ kilo litre trucks of fuel per day (805 kilo litres) during the RTS cycle. The vendor agrees to supply 100x35 kilo litre trucks of fuel given some specified CPLT. The yearly RTS cycle has a minimum demand of approximately 200 kilo litres per day and a maximum of 800 kilo litres per day. The contingency demand is estimated to be 3500 kilo litres per day and lasts for 8 weeks, as is shown in Figure 3. The vendor agrees that it can supply the surge rate in a CPLT of 69 weeks. The warning time of the contingency is between 1 to 8 weeks.

Figure 4 graphs the initial inventory holdings and the reserve stock required to meet both RTS and contingency demands, and how they are influenced by the warning or preparedness time and the CPLT. One can observe that when the warning time is sufficiently large, the actual infrastructure capacity required is considerably smaller than for shorter warning times, as a throughput-based or distribution-based logistics 
Calbert et al., Military inventory capacity and stock planning with surge, warning time and supplier constraints

solution can be found. When the warning time is small compared to the CPLT, a stockpile bases logistics infrastructure solution must be found.
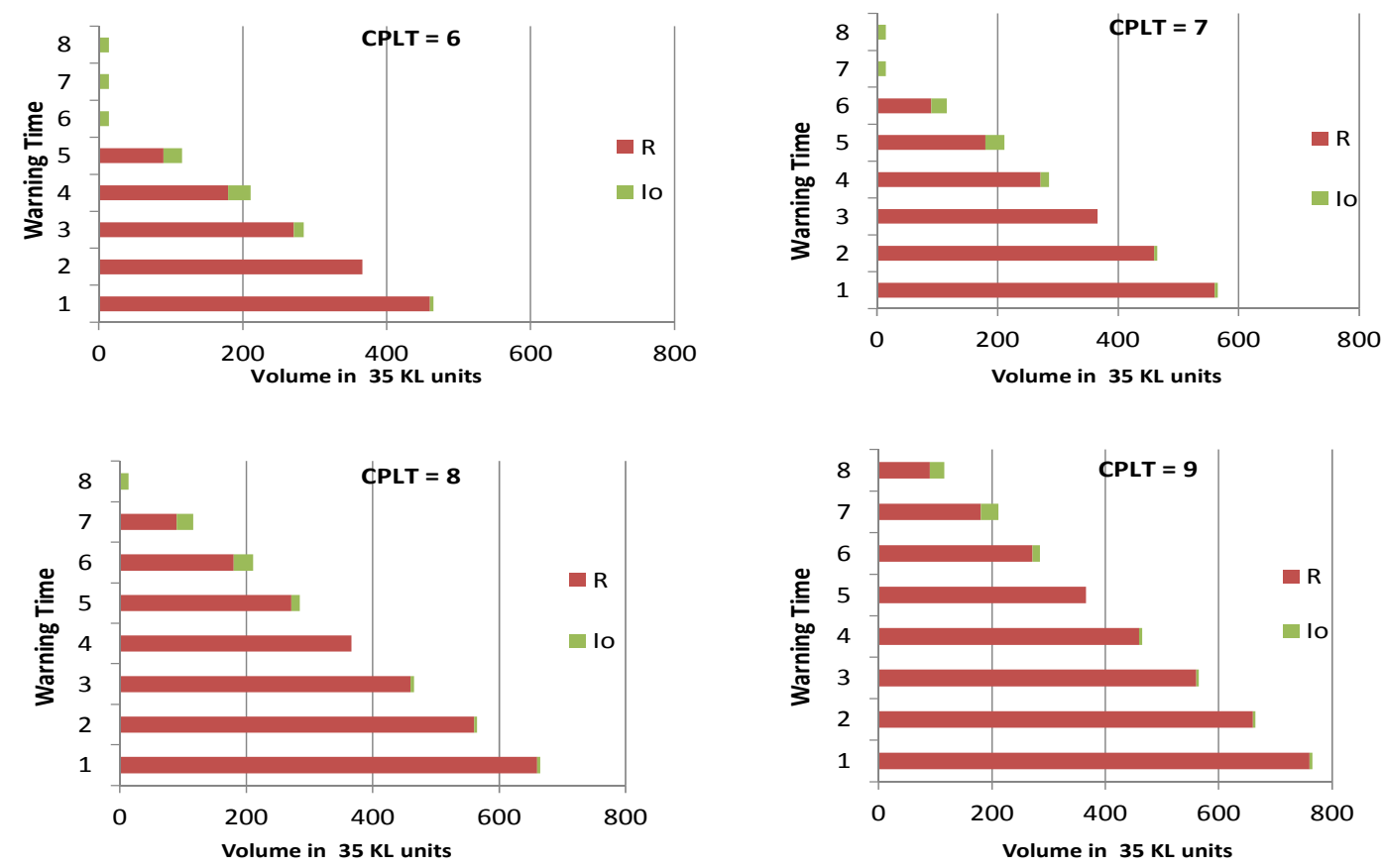

Figure 4. Initial inventory and reserve stocks as a function of both the CPLT and warning time.

\section{RESILIENCE AND STOCHASTICITY}

While we have considered minimising the capacity, which minimises the cost, it is important to factor in resilience to supply chain uncertainties. One approach to doing this is to ensure the bottom of the inventory cycle is non-zero and includes some safety stock, analogous to re-order point approaches to inventory management. This is easily done by setting the constraint $I_{t}^{\text {bottom }}(\tau) \geq k \sigma_{\tau}$ where $\sigma_{\tau}$ is the standard deviation of the demand time series, given the work-up, contingency starts at time $\tau$. The factor $k$ determines that resilience of the system, as in standard inventory theory. Typically, if the underlying demand distribution is assumed to be Gaussian, if $k=2$, then the probability of not stocking out in percentage terms is $97.7 \%$ (Blumenfeld, 2001).

Another approach to the development of resilience is to apply the methods of stochastic linear programming (SLP) (Sharpiro and Philpott, 2007). SLP is now being used to look at supply chains in the oil industry (Dempster et al. 2000). In the discipline of SLP, two sets of decisions are considered in what is termed twostage programming. The first sets of decisions, which in the context of this paper are the decisions on infrastructure capacity, are made first. A stochastic event occurs which leads to a second set of decisions, which in the context of the paper are the fuel purchasing decisions. The stochastic events are called scenarios in the parlance of SLP. Each scenario is a time sequence of fuel demands, which may or may not include contingency demands with associated warning and contingency provisioning lead times. If we add the superscript $F_{j}, j=1, \cdots, N$ to specify a particular scenario, and $\mathbf{x}^{F_{j}}, \mathbf{s}^{F_{j}}$ to be the decision vectors for nominal and surge supply in this scenario then the SLP structure is to minimise the capacity

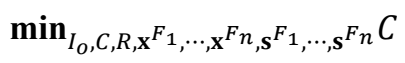

where each scenario induces a set of constraints simular to equation (2). The set of scenarios $N$ may be very large or infinite. Here Monte-Carlo simulation is used to sample a subset of scenarios and apply what is termed the sample average approximation algorithm (SAA). For a subset of $M \ll N$ scenarios, the number of constraints in the SLP will be $2 M(T(T+1)+S P)+3$. Clearly, for models over a yearly RTS cycle, and multiple samples, the SLP will have large numbers of constraints, testing computational feasibility. 
Calbert et al., Military inventory capacity and stock planning with surge, warning time and supplier constraints

Nonetheless, SLP has the advantage of modelling non-Gaussian demand statistics and importantly uncertainties in warning time. The application of SLP methods is an important current area of research.

\section{DISCUSSION AND CONCLUSIONS}

In this paper, we have modelled linked the link between Defence preparedness or warning times and the implications for Defence infrastructure, inventory holdings and reserve stocks. In an era where cost optimisation is important, with this analysis, logisticians have the opportunity to carefully examine their stockholdings. Often rules of thumb are used by the military to make stockholding decisions. Keeping so many days of supply at average RTS demand levels is a simple heuristic. Nonetheless, with the era of modern data analytics, we can apply the optimisation methods described, to see whether the heuristic applied is sufficient or there is a potential shortfall for some scenarios.

Research in the application of optimisation for Defence infrastructure has considerable scope for expansion, to include stochasticity, combining demand forecasting and optimisation, item perishability, and importantly networking. One important conclusion to be made is that Defence planners do not need to necessarily store all logistics items required to meet a scenario, if the warning time is large and the supply chain reactive.

\section{ACKNOWLEDGMENTS}

The authors acknowledge Andrew Wraith of Spectrum Energy, who discussed the importance of linear programming in petroleum distribution planning.

\section{REFERENCES}

Bixby, R. (2010). Solving real world linear programs: a decade and more of progress. Operations Research, $1-25$.

Blumenfeld, D. (2001). Operations Research Calculations Handbook. CRC Press.

Defence, (2004). Australian Defence Doctrine Publication, Preparedness and Mobilisation. http://www.defence.gov.au/adfwc/Documents/DoctrineLibrary/ADDP/ADDP_00_2_preparedness_and_m obilisation.pdf

Dempster, M, Hicks Pedron, N., Medova, E. Scott, J. and Sembos, A. (2000). Planning logistics operations in the oil industry. Journal of the Operational Research Society, 51, 1271-1288.

Sharpiro, A., and Philpott, A. (2007). A tutorial on stochastic dynamic programming. http:/www.cse.iitd.ernet.in/ Naveen/courses/CSL866/TutorialSP.pdf

Stevens, A., and Ingram, H.J. (2013). Reframing the Defence Discourse: Australia's 'black swan' and its implications for preparedness and mobilisation. Australian Defence Force Journal 190, 30-43. 